\title{
Implementation of ipilimumab therapy in a private practice oncology group: overcoming start-up and reimbursement issues related to expensive new cancer drugs
}

\author{
Wolfram E Samlowski, MD; Matthew J Williams, PharmD; Jessica L Weger, CPC, CRCR; \\ Paulette Kissel; Sherry Neal, RN; Merle Witter, CCRP; and Suzanne Samlowski, M Arch \\ Comprehensive Cancer Centers, Las Vegas, Nevada
}

\begin{abstract}
The monoclonal antibody ipilimumab was the first treatment in more than 30 years to improve long-term survival in metastatic melanoma patients. Offering expensive ipilimumab treatment presented significant business challenges and potential financial risks for our private oncology practice and for patients because of the high acquisition cost of this agent. There was initial uncertainty about the willingness of insurance companies to reimburse for this new drug based on previous experiences in our practice with other expensive new drugs. Here we describe how our multiphysician practice methodically introduced ipilimumab treatment into the practice. Structured communication between the clinical, pharmacy, and business components of the practice proved to be critical. The financial counselor, pharmacist, primary nurse, and physician coordinated each patient's financial and medical information. Efficient drug ordering practices were devised. Our practice made an initial calculation of how much debt was reasonable as a test of reimbursement mechanisms and set an initial limit of 3 patients to be treated with ipilimumab. The billing specialists and pharmacist closely monitored pending claims on a weekly basis to stay aware of potential problems in claims processing. Due to the initial success of this approach, the number of patients being treated has steadily increased. Our experience allowed us to establish a model for how the use of new and expensive cancer therapies can be implemented in a private practice clinic without causing excessive financial risk. Our practice was able to analyze the financial viability of a new treatment that offered significant benefit for patients within 6 months of starting the first pilot patient.
\end{abstract}

$\mathrm{F}$ or decades, the median survival of metastatic melanoma patients was less than 7 months, with only $15 \%$ of patients achieving 1 -year survival, regardless of treatment. ${ }^{1}$ Ipilimumab is a fully human monoclonal antibody $(\mathrm{mAb})$ that reverses tumor-induced immunosuppression by blocking the interaction of CTLA-4 with its ligands, CD80/CD86., ${ }^{2,3}$ This agent significantly improved survival in metastatic melanoma patients. ${ }^{4}$ Ipilimumab was approved by the US Food and Drug Administration (FDA) in 2011 for treatment of unresectable and metastatic melanoma. A recent update of long-term follow-up of ipilimumabtreated patients has demonstrated a survival plateau of $18 \%-22 \%$ between 3-10 years after treatment., 5

Based on this data, we wanted to offer ipilimumab therapy in our multiphysician oncology practice. Virtually none of the physicians in our practice had any prior experience with the agent. Only 1 of 34 physicians had extensive prior experience with the $\mathrm{mAb},{ }^{7}$ and was the major advocate. The high drug purchase cost, and uncertainties about billing mechanisms and reimbursement were concerns within the practice. Insurance copayments from patients can also result in significant financial costs and duress to patients with cancer. In some cases, these represent as much as $20 \%$ of the purchase cost of the medication. We describe here the challenges and success of implementing ipilimumab therapy through careful planning and active involvement of medical, administrative, and support staff. Lessons learned from this process have subsequently been used in our practice to evaluate and implement other new, effective, and expensive medications.

\section{Methods \\ Local context \\ Our practice administration is supportive of bring-}

Accepted for publication March 8, 2016. Correspondence: Wolfram Samlowski, MD; wsamlowski1 @gmail.com. Disclosures: Dr Samlowski - advisory boards: Novartis, Pfizer; speakers bureaus: Bristol-Myers Squibb, Merck, Novartis, Pfizer. The remaining authors report no disclosures or conflicts of interest. JCSO 2016;14:244-248. (02016 Frontline Medical Communications. doi: 10.12788/jcso.0257. 
ing new "breakthrough" agents into the group to satisfy unmet medical needs as long as the agents don't create a financial liability to the practice. There were initially concerns in the practice about insurance reimbursement for ipilimumab and how quickly insurance payors would reimburse drug purchases because of the high initial drug acquisition costs. Previous adverse reimbursement experiences with another contemporary high-cost biologic agents had made our practice leadership very cautious.

The onus was on interested physicians to convince others in the practice of the feasibility of successfully introducing an expensive but potentially useful new agent into the clinic. To better understand reimbursement issues, regional oncology practices were surveyed to gather information on ipilimumab billing and reimbursement. Within a 5-state region, only 1 oncology practice (in Reno, Nevada) had any prior insurance reimbursement experience. That practice had treated 3 patients and had received reimbursement for all 3, suggesting a high likelihood of in-state reimbursement of ipilimumab by both private insurance and Medicare. This information was reassuring and suggested that ipilimumab could prove financially viable.

\section{Method for implementation}

It became clear that a number of processes in multiple departments of the practice needed to be coordinated, to assure that a patient's medical and financial evaluations, insurance approval, drug acquisition, treatment, and posttreatment monitoring would take place in a seamless and timely manner (Figure 1).

The physician with the most ipilimumab treatment experience was selected to enroll 3 patients as a feasibility pilot. This physician identified 3 metastatic melanoma patients as candidates for ipilimumab treatment, and a clinical eligibility assessment was performed. The risks, benefits, and schedule for ipilimumab treatment were discussed with each patient.

The physician was also responsible for placing treatment orders into the electronic medical record (EMR). His primary nurse was assigned the responsibility for alerting clinical, pharmacy, and business components of the practice by e-mail about a potential ipilimumab patient. The billing specialist obtained pre-authorization from the patient's insurance company, using the treatment orders, diagnostic codes, and supporting documentation from the EMRs. Verification of insurance pre-approval was required before any ipilimumab purchase to minimize any later reimbursement issues.

The primary nurse also was charged with notifying the financial counselor about the details of each selected patient. The counselor explained potential financial copayments to the patient and family and helped them complete paperwork for the BMS Oncology Access Support Program to minimize out-of-pocket costs and provide copayment cov-

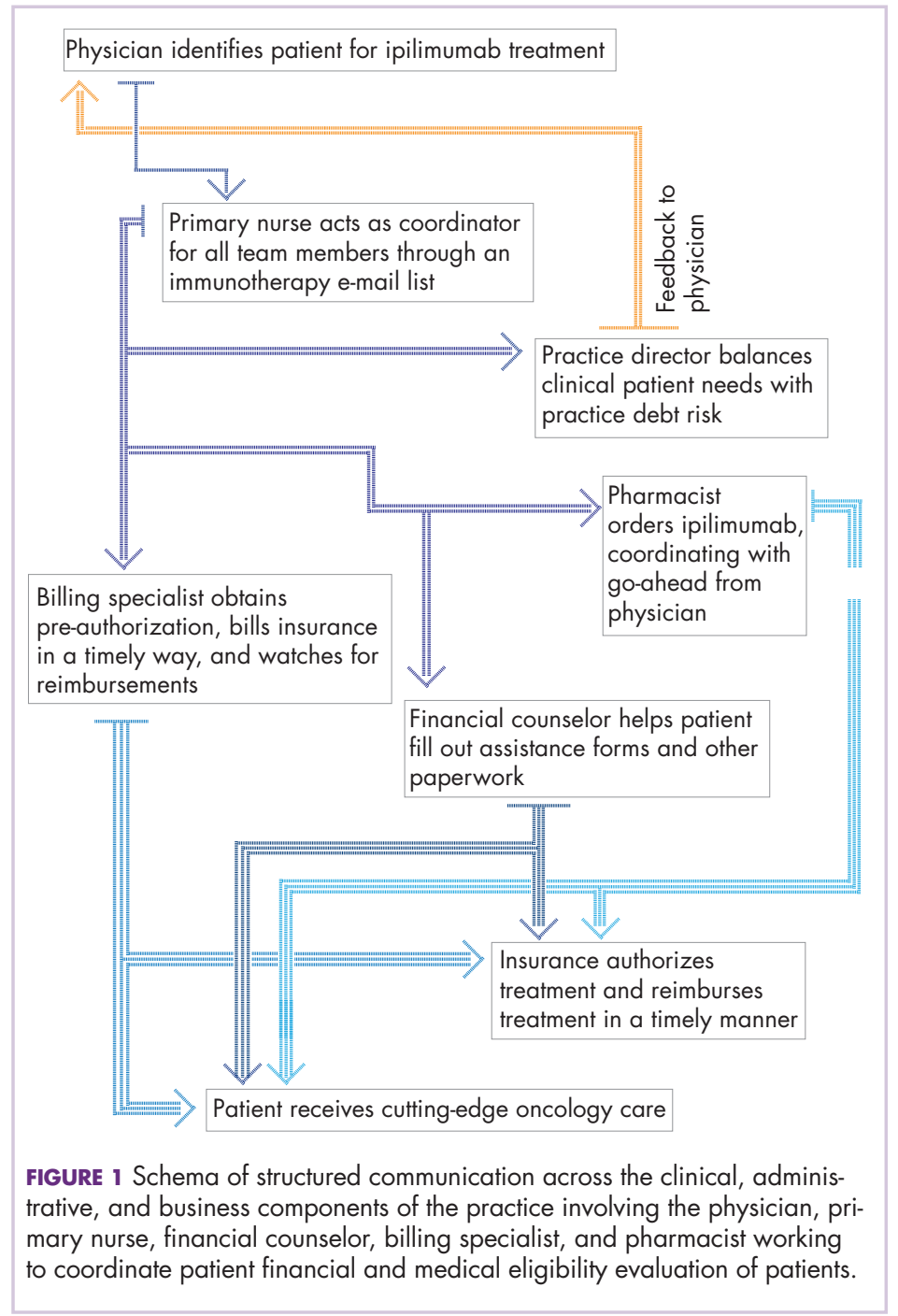

erage (especially for patients with high copayment obligations). The support program is designed to assist with prior authorization with insurances, appeal denied insurance claims, provide billing codes, provide copay assistance for underinsured patients, and assist in identifying charitable foundation support (for Medicare patients; http://www. bmsaccesssupport.bmscustomerconnect.com).

Applying for this program on behalf of each ipilimumab patient initially placed an extra burden on the financial counselor. With experience, the application process became more efficient and streamlined for the financial counselor. BMS (Bristol-Myers Squibb, the maker of ipilimumab) provided a specific point of contact for our practice, which further improved the time for application approval to 24-72 hours.

The physician re-evaluated the patient within a week of the planned start of treatment to be sure the patient was stable enough to begin ipilimumab therapy. With the physician's final approval, the pharmacist placed the drug order. 
When ipilimumab arrived in the pharmacy, the patient was scheduled into the infusion room for treatment. This "just-in-time" approach shortened any potential time lag between drug purchase, drug infusion, billing, and reimbursement, which would otherwise cause a large debt to the practice. Because of the high initial drug purchase cost - often $\$ 24,000-\$ 36,000$ per dose of ipilimumab (Table), any lengthy lag in reimbursement would have had a negative impact on the financial viability of this agent.

\section{Setting a limit on financial risk}

The practice physicians and executive director set an initial limit of 3 patients who could be treated with ipilimumab as a feasibility test. The billing specialists and pharmacist closely monitored all pending claims on a weekly basis to stay aware of problems in processing claims. After the initial 3 patients completed treatment, a financial assessment was performed and presented to the practice. During this 4-month interval, 3 additional ipilimumab-eligible patients were treated with alternate first-line therapy. Each of these patients subsequently received ipilimumab as second-line therapy after disease progression.

\section{Results}

\section{Clinical results of ipilimumab therapy}

The initial follow-up of billing and insurance reimbursement for the first 3 pilot patients (treated during July 2011-November 2011) was reviewed by the practice physicians in January 2012. Because of the careful process we had used, the experiment proved to be a financial success because insurance reimbursement was obtained within 3 months for all 3 patients. With the ongoing success of the monitoring process that was implemented, the number of patients able to be treated at any one time was steadily expanded.

In the first year, the BMS support program provided $\$ 15,276$ in assistance to 4 of our patients. Charitable foundations paid $\$ 42,548$ for copayment expenses of 2 additional Medicare-insured patients, who did not qualify for the support program. It should be noted that Medicare patients are not eligible for most pharmaceutical company drug-assistance programs.

Despite the most diligent efforts, insurance preapproval is never a guarantee of reimbursement. As a result of our proactive approach, only 3 of 108 doses of ipilimumab in the initial 31 patients were unreimbursed by insurance companies. All 3 denied doses were for 1 patient, who inexplicably had modified her insurance plan after insurance preapproval of her first ipilimumab dose. The purchase cost for these 3 doses was eventually reimbursed through a separate BMS unreimbursed drug cost repayment program. One additional dose of ipilimumab ordered for a very heavy patient was underreimbursed by $\$ 1800$, even though his insurance company had appropriately reimbursed his first 3 doses based on actual body weight. In the first year of using the drug in our practice, 31 patients received 108 doses of ipilimumab, with an average profit to the practice that exceeded the average sales price (ASP) by more than $6 \%$.

The survival of melanoma patients following ipilimumab

\begin{tabular}{|c|c|c|c|c|c|c|c|}
\hline \multirow[b]{2}{*}{ Agent } & \multirow{2}{*}{$\begin{array}{c}\text { RECIST }^{c} \\
\text { response } \\
\text { rate, } \%\end{array}$} & \multirow{2}{*}{$\begin{array}{l}\text { Median } \\
\text { PFS, mo }\end{array}$} & \multicolumn{3}{|c|}{ Survival, \% } & \multicolumn{2}{|c|}{ Estimated cost, US\$ ${ }^{\mathrm{d}}$} \\
\hline & & & 1 year & 2 year & 3 year & 3 mo & $12 \mathrm{mo}$ \\
\hline Temozolomide $^{8}$ & 13.5 & 1.9 & 30 & 20 & $\sim 10$ & 11,000 & 43,000 \\
\hline Carboplatin + paclitaxel ${ }^{9 e}$ & 18.2 & 4.2 & 48 & 25 & 18 & 8,000 & 33,000 \\
\hline High-dose IL-2 ${ }^{10 f}$ & 28 & NA & 59 & 41 & 31 & 300,000 & 600,000 \\
\hline Dabrafenib ${ }^{12 g}$ & 51 & 8.8 & 68 & 42 & NA & 26,000 & 106,000 \\
\hline Dabrafenib + trametinib ${ }^{12 g}$ & 67 & 9.3 & 74 & 51 & NA & 57,000 & 229,000 \\
\hline Ipilimumab 6 & 10 & 11 & 50 & 35 & 25 & 133,000 & 133,000 \\
\hline Pembrolizumab ${ }^{13}$ & 32.9 & 4.2 & 68.4 & NA & NA & 34,000 & 138,000 \\
\hline
\end{tabular}

IL-2, interleukin 2; NA, not applicable; PFS, progression-free survival; RECIST, Response Evaluation Criteria in Solid Tumors

${ }^{\circ}$ Cost data is provided for an estimated 3-month treatment interval, because this approximates the duration of 1 cycle (4 doses) of ipilimumab therapy. Because the average survival of ipilimumab-treated patients is close to 12 months, drug cost information spanning this interval is also provided. An important caveat for this table is that the studies cited had variable entry requirements that could alter outcome and therefore they are not strictly comparable. This table is intended to provide a sense of the relative costs of agents and relevant clinical benchmarks. It is not intended to imply direct comparison in head-to-head trials. It should be noted that survival and costs are also likely to be increased by subsequent use of additional active treatments. ${ }^{b}$ Drug costs were estimated from published sources based on $80 \mathrm{~kg}$ weight and 2.0 m body surface area, from 2015 published references. ${ }^{2} \mathrm{RE}-$ CIST response criteria may underestimate treatment response to immunotherapy. ddollar amounts are rounded to the nearest 1000 . eNot counting infusion room costs or the costs of needed premedications. fHighly selected patient eligible for high-dose IL-2. Drug cost estimated based on our clinical experience using 600,000 units/kg every $8 \mathrm{~h}$ for days $1-5$ and $11-15$, for 2 cycles and ICU hospitalization costs. 9Patients with BRAF V600 E or K mutations only were eligible. 


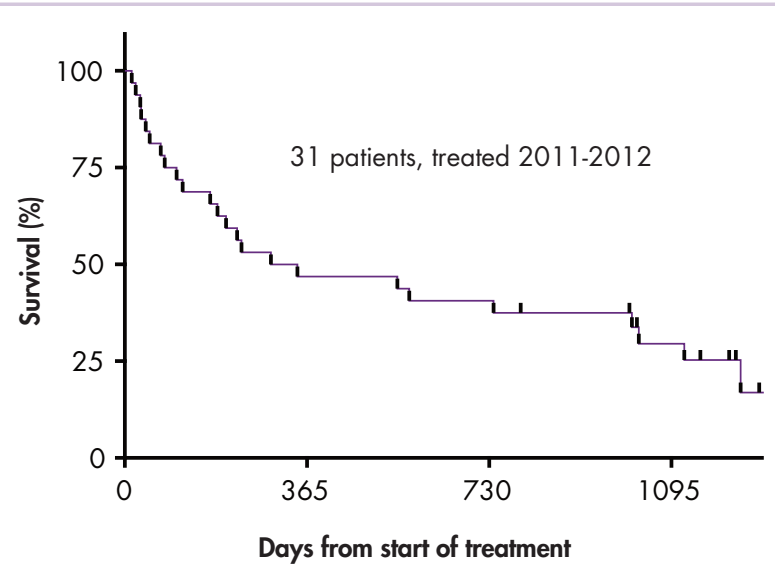

FIGURE 2 Survival of the initial 31 metastatic melanoma patients treated with ipilimumab. Median survival of ipilimumab treated patients was 793 days, including 23 skin melanomas, 1 unknown primary, 1 ocular, 1 acral, 3 desmoplastic, and 3 mucosal melanoma patients. This cohort of patients was unselected and had adverse features. Only 12 patients were previously untreated, 13 patients had 2 or more prior treatment failures. Eight patients had BRAF V600E mutations, 1 had an NRAS mutation. Treated brain metastases were present in 15 patients, and 2 additional patients had significant spinal cord involvement.

treatment in our practice is shown in Figure 2. Among the first 31 patients, many had adverse prognostic features, including 15 patients with brain metastases. Overall, $4(13 \%)$ patients eventually had a complete response, 3 (10\%) had a partial response, and $3(10 \%)$ achieved longterm stable disease (32.3\%). Twenty-one patients eventually had disease progression (67.7\%). The median survival of the entire patient cohort was 793 days (about 2.2 years). These encouraging clinical results show why it is desirable to expand the use of ipilimumab and other new agents into private practice settings. Our practice treated more than 110 patients with ipilimumab during 2011-2015.

\section{Discussion}

Offering ipilimumab treatment, as well as other expensive new drugs, presents significant business challenges and financial risks for private oncology practices. With ipilimumab, there was an initial reluctance in our practice to take on the financial risk associated with it. There also was initially uncertainty about the willingness of insurance companies to pay for it. However, given the precautions we take, insurance reimbursement has turned out to not be a financial obstacle to using it in the practice.

To make ipilimumab therapy viable, we organized systematic, structured communication between the clinical, pharmacy, and business components of the practice to track reimbursement in real time for each patient. The financial counselor, pharmacist, primary nurse, and physician coordinated each patient's financial and medical information. Efficient "just-in-time" drug ordering prac- tices were implemented. The billing specialists and pharmacist closely monitored pending claims on a weekly basis to stay aware of potential problems in claims processing. Because of the initial success of that approach, the number of patients being treated with this agent in our practice has steadily increased. Our experience with ipilimumab helped us establish a model for how all other new and expensive cancer therapies are currently being evaluated and implemented in our private practice. Over a 6-month span, our practice was able to establish the financial viability of a new treatment that offers significant clinical benefit for patients.

Management of the increasing costs of medical care, especially related to new, expensive cancer medications is a growing national concern. Ironically, this pressure comes at a time, when after many decades of futility, these new treatments are improving clinical responses and survival across many cancer types. Despite clear demonstration of long-term survival in more than $20 \%$ of metastatic melanoma patients treated with ipilimumab, there is increasing push-back from capitated, managed care, and HMO (health maintenance organization) plans to minimize or prevent use of checkpoint inhibitors such as ipilimumab to cut costs. The very high initial acquisition costs for ipilimumab and other new immunotherapy drugs are a significant financial risk to small- and moderate-sized private practices. Managing the "float" or time from drug purchase until reimbursement is crucial for financial viability of a new drug.

To provide some background for a discussion of drug purchase costs for melanoma treatment agents, we have estimated the acquisition cost and provided references to published data concerning the magnitude of benefit of current agents (Table). As a reference, relative drug acquisition costs and outcome information for other contemporary melanoma treatments is provided. The $\$ 96,000-120,000$ drug purchase cost for 1 cycle of ipilimumab (4 doses at 3 $\mathrm{mg} / \mathrm{kg}$ ) is balanced against a median survival of 2.2 years in our small series. With long-term follow-up, about $20 \%$ of ipilimumab-treated patients have achieved long-term (3-10 year) survival. ${ }^{6}$

We continually analyze reimbursement patterns for ipilimumab and other new breakthrough drugs in our practice. The practice was generally reimbursed for ipilimumab purchases by insurance within 6-8 weeks. Outright denial of payment following preliminary approval occurred only once (for 3 unpaid doses of ipilimumab for 1 patient). After a failed year-long appeals process, our cost for purchasing the drug was eventually reimbursed by BMS. The cost for 1 dose of ipilimumab in a separate patient was underpaid and eventually written off.

Medicare drug reimbursement has been especially inconsistent because reimbursement for manufacturer price increases are adjusted only every 6 months based on the ASP formula. For example, in November 2014, 
ipilimumab reimbursemement for Medicare patients dropped to ASP plus 1.6\% per dose because of price increases by the manufacturer. Profitability was eventually restored by Medicare reimbursement adjustment for ASP 6 months later, in mid-2015. The 2012 federal budget "sequester" of Medicare drug reimbursement funds also had a negative impact on the use of newer cancer treatments in Medicare patients due to delayed treatment reimbursement. ${ }^{15}$

The sharing of treatment experiences using expensive new medications among physicians within and between practices will help establish the feasibility of newer treatment options and provide melanoma patients improved opportunities for survival. Evidence-based care guidelines and demonstra-

\section{References}

1. Korn EL, Liu PY, Lee SJ, et al. Meta-analysis of phase II cooperative group trials in metastatic stage IV melanoma to determine progression-free and overall survival benchmarks for future phase II trials. J Clin Oncol. 2008;26:527-534.

2. Gajewski TF. Identifying and overcoming immune resistance mechanisms in the melanoma tumor microenvironment. Clin Cancer Res. 2006;12:2326s-2330s.

3. Melero I, Hervas-Stubbs S, Glennie M, Pardoll DM, Chen L. Immunostimulatory monoclonal antibodies for cancer therapy. Nat Rev Cancer. 2007;7:95-106.

4. Hodi FS, O'Day SJ, McDermott DF, et al. Improved survival with ipilimumab in patients with metastatic melanoma. N Engl J Med. 2010;363:711-723.

5. Maio M, Grob JJ, Aamdal S, et al. Five-year survival rates for treatment-naive patients with advanced melanoma who received ipilimumab plus dacarbazine in a phase III trial. J Clin Oncol. 2015;33:1191-1196.

6. Schadendorf D, Hodi FS, Robert C, et al. Pooled analysis of longterm survival data from phase II and phase III trials of ipilimumab in unresectable or metastatic melanoma. J Clin Oncol. Published online February 9, 2015. doi: 10.1200/JCO.2014.56.2736.

7. Hersh EM, O'Day SJ, Powderly J, et al. A phase II multicenter study of ipilimumab with or without dacarbazine in chemotherapy-naive patients with advanced melanoma. Invest New Drugs. 2011;29:489-498.

8. Middleton MR, Grob JJ, Aaronson N, et al. Randomized phase tion of substantial improvements in patient survival will help oncologists counteract restrictive trends and allow practices to successfully negotiate with third-party payers to provide the best possible cancer care to their patients.

\section{Acknowledgment}

The authors acknowledge the assistance of our infusion room and nursing staff, who helped treat these patients.

\section{Disclaimer}

This publication represents the personal opinion of the authors and not necessarily that of our medical oncology practice, its suppliers, or BristolMyers Squibb, the maker of ipilimumab. BMS did not influence the content of the manuscript, nor did the authors receive financial compensation for authoring the manuscript.

III study of temozolomide versus dacarbazine in the treatment of patients with advanced metastatic malignant melanoma. J Clin Oncol. 2000;18:158-166.

9. Flaherty KT, Lee SJ, Zhao F, et al. Phase III trial of carboplatin and paclitaxel with or without sorafenib in metastatic melanoma. J Clin Oncol. 2013;31:373-379.

10. Payne R, Glenn L, Hoen H, et al. Durable responses and reversible toxicity of high-dose interleukin-2 treatment of melanoma and renal cancer in a Community Hospital Biotherapy Program. http:// www.ncbi.nlm.nih.gov/pmc/articles/PMC4030280/.J Immunother Cancer. Published online May 14, 2014. Accessed May 19, 2016.

11. Sosman JA, Kim KB, Schuchter L, et al. Survival in BRAF V600mutant advanced melanoma treated with vemurafenib. N Engl J Med. 2012;366:707-714.

12. Long GV, Stroyakovskiy D, Gogas H, et al. Dabrafenib and trametinib versus dabrafenib and placebo for Val600 BRAF-mutant melanoma: a multicentre, double-blind, phase 3 randomised controlled trial. Lancet. 2015;386:444-451.

13. Robert C, Schachter J, Long GV, et al. Pembrolizumab versus ipilimumab in advanced melanoma. N Engl J Med. 2015;372:2521-2532.

14. Robert C, Long GV, Brady B, et al. Nivolumab in previously untreated melanoma without braf mutation. N Engl J Med. 2014.

15. Anonymous. What does sequestration mean to physicians and hospitals? [Internet publication]. 2014; http://www.medicarenewsgroup. $\mathrm{com} /$ news/medicare-faqs/individual-faq? faqId $=2 \mathrm{ec} 7 \mathrm{~b} 6 \mathrm{bb}-\mathrm{c} 68 \mathrm{~b}-$ 433e-830e-035b9d930e4d. Accessed 5/19/16, 2016. 